\title{
ENTREVISTA CON RAFAEL CUEVAS MOLINA: ESTADOS-NACIÓN, IDENTIDADES E INTEGRACIÓN EN AMÉRICA LATINA
}

\section{INTERVIEW WITH RAFAEL CUEVAS MOLINA. NATION- STATES, IDENTITIES AND INTEGRATION IN LATIN AMERICA}

Fecha de recepción: 03/07/2018

Fecha de aceptación: 17/07/2018

Palabras clave: entrevista; identidades nacionales; integración; América Latina; Estadonación; Rafael Cuevas Molina.

Keywords: Interview; National Identity; Integration; Latin America; State; Rafael Cuevas Molina.

\section{Presentación}

Rafael Cuevas Molina (Guatemala, 1954)* es un destacado historiador, escritor y artista plástico. Licenciado en filosofía y magíster en historia cuyos estudios ha llevado a cabo en Rumanía y Costa Rica. Doctor en Historia por la Universidad de La Habana, Cuba. Es catedrático, investigador y profesor en el Instituto de Estudios Latinoamericanos (IDELA), adscrito a la Facultad de Filosofía y Letras de la Universidad Nacional (UNA), Costa Rica. Dirige el Suplemento Cultural, ${ }^{1}$ publicación conjunta del IDELA y del Programa Identidad, Cultura, Arte y Tecnología (ICAT) del Centro de Investigación, Docencia y Extensión Artística (CIDEA) de la UNA, así como Con Nuestra América, órgano difusor de la Asociación por la Unidad de Nuestra América

\footnotetext{
* Guatemalteco. Doctor en Historia por la Universidad de La Habana, Cuba. Profesor e investigador del Instituto de Estudios Latinoamericanos (IDELA), de la Universidad Nacional (UNA), Costa Rica. Correo electrónico: rafael.cuevas.molina@una.ac.cr

${ }^{1}$ Véase: http://www.icat.una.ac.cr/suplemento cultural/index.php/en/
} 
(AUNA-Costa Rica). ${ }^{2}$ Connotado investigador y especialista en problemáticas y temas referentes a la construcción de identidades culturales y nacionales en América Latina y, especialmente, de Centroamérica, ha publicado numerosos artículos, libros, ensayos, novelas, poemarios y ha realizado exposiciones de sus obras plásticas en Costa Rica, Guatemala y Cuba. La siguiente tan solo representa una muestra de su extensa producción interdisciplinaria: ${ }^{3}$

- 300 (Heredia, Costa Rica: EUNA, 2011).

- El punto sobre la: políticas culturales en Costa Rica (1948-1990) (San José, Costa Rica: Ministerio de Cultura, Juventud y Deportes, 1995).

- De Banana Republics a repúblicas maquileras. La cultura en Centroamérica en tiempos de globalización neoliberal (1990-2010) (San José, Costa Rica: EUNED, 2012).

- Sandino y la intelectualidad costarricense. Nacionalismo antiimperialista en Nicaragua y Costa Rica (1927-1934) (San José, Costa Rica: EUNED, 2008)

- Cultura y política en Costa Rica: entrevistas a protagonistas de la política cultural en la segunda mitad del siglo XX, Colección Historia Cultural de Costa Rica No 5 (San José, Costa Rica: EUNED, 2006).

- en coautoría con Andrés Mora Ramírez, Buscando el futuro. Crisis civilizatoria y posneoliberalismo en América Latina (San José, Costa Rica: EUNED, 2015).

\footnotetext{
${ }^{2}$ Véase: https://connuestraamerica.blogspot.com/

3 Para una consulta pormenorizada de la amplia obra del autor entrevistado, véase: http://una$\underline{\text { cr.academia.edu/RCuevasMolina }}$
} 


\section{Entrevista}

¿Qué tan vigentes son hoy los Estados-nación como estructuras de identidades $y$ cómo incide esta situación en las actuales manifestaciones de xenofobia?

Los estados-nación se encuentran en un momento de cuestionamiento de su vigencia por los procesos de globalización y seguramente hacia el futuro, tendencialmente, esta situación se irá acentuando. Digo tendencialmente porque es posible que haya momentos en los que tal situación se revierta momentáneamente, pero a largo plazo, seguramente darán paso a nuevas formas de organización macrosocial.

Dicho lo anterior, debe aclararse que aunque la tendencia es a una pérdida paulatina de su vigencia, eso no implica que en el mundo contemporáneo las naciones no sigan cumpliendo funciones muy importantes, como de hecho las cumplen. En primer lugar, las naciones siguen siendo el principal referente identitario de grandes grupos sociales enmarcados en las fronteras estatales, lo que quiere decir que siguen otorgando sentido de pertenencia y rasgos de personalidad social diferenciadores.

Estas identidades colectivas son, en muy buena medida, construcciones modernas, lo que quiere decir que son producto de cierta ingeniería social impulsada por grupos sociales interesados en conjuntar voluntades que legitimen un determinado proyecto político. En América Latina, el proceso de construcción del nacionalismo, aunque tiene antecedentes lejanos en el período colonial, se produce en el siglo XIX, especialmente en su segunda mitad cuando los liberales se ven en la necesidad de legitimar el proyecto oligárquico que impulsaban. Se dan entonces a la tarea de concebir las diferencias nacionales en un universo regional en el que prevalecían los elementos que abonaban a la 
similitud: el idioma, la religión, las formas de gobierno, costumbres y tradiciones.

Un aspecto central del nacionalismo es, por lo tanto, construir la diferencia, lo que significa identificar un(as/os) otro(a/s) del cual separarse y distanciarse o, también, con quien identificarse. Este segundo aspecto es muy importante en América Latina, que tiene sus raíces en el período colonial, cuando se estructuró un tipo de mentalidad según la cual el modelo a seguir se encontraba en el centro del sistema, en este caso en España, por lo que se creó lo que podríamos llamar una mentalidad colonial, que consiste en imitar por considerarlo mejor o más adelantado o más acabado, lo que sucede o se hace en el centro. Ese centro a imitar ha cambiado con el tiempo. Primero fue España, luego París, más tarde Nueva York y así sucesivamente.

Como ya indicamos, paralelo al modelo imitable existe otro, negativo, concentrador de lo que no se quiere ser, al que se le achacan e "inventan" rasgos rechazables y que reafirman lo que se considera positivo y que se concentraría en el yo social nacional propio. La xenofobia se orienta generalmente hacia ese alter ego que se repele y con el que no se quisiera estar en contacto porque "contamina". Mientras ese otro se encuentre lejos y no interfiera en la vida cotidiana puede tolerarse, pero entrar en contacto con él causa rechazo en diferentes grados.

Esos sentimientos de diferencia colectiva unifican al grupo y son fácilmente manipulables políticamente. Exacerbar los sentimientos de exclusividad nacional subyacentes, muchas veces adormecidos o inocuos pero siempre presentes es un recurso de relativa fácil invocación que obnubila los sentidos y se sobrepone sobre otros.

Todo lo anterior muestra la vigencia que sigue teniendo el nacionalismo en nuestros días y no cabe duda que, aunque tendencialmente la nación se 
encuentra en declive, no solo tiene para rato sino que juega un papel central en procesos socio políticos centrales de la contemporaneidad.

\section{Nos interesa conocer su opinión sobre cómo se ha tratado este tema en Costa Rica.}

Como cualquier otro estado-nación latinoamericano, en Costa Rica la construcción de la identidad nacional hegemónica de origen liberal, pero como cualquier identidad -individual o social- en constante cambio, se afianza sobre la diferencia que en este caso se expresa como excepcionalidad.

El concepto y el sentimiento de excepcionalidad se expresa de diferentes formas, desde las más básicas -el país escogido y protegido por Dios, por ejemplo- hasta las más sofisticadas, con empaque "científico"- pero que tienden a reforzar la separación del resto, en este caso Centroamérica con Nicaragua en primer lugar. Un ejemplo de este tipo es la discusión renovada cada año de la fecha en la cual debería de conmemorarse la independencia, en la cual se argumenta que en Costa Rica no debería ser en setiembre sino en octubre. Otro es el cambio que sufrió la forma de entender y nombrar lo que hoy se conoce como Campaña Nacional que refiere a los hechos de 1856 y que antes era conocida como Campaña Centroamericana.

Ese sentimiento de excepcionalidad es de superioridad sobre el resto y emana de muchos lugares distintos. En Costa Rica va de una supuesta mayor blancura de sus habitantes en relación con los del resto del istmo y que lleva implícita la idea eurocéntrica de que ser "blanco" es mejor que ser indio, negro o mestizo "oscuro", hasta considerar que su(s) clase(s) dominante(s) es más inteligente que la del resto del istmo, como si se tratara de razones genéticas; este anclaje en lo genético se traslada a otros factores que construyen el andamiaje de la excepcionalidad, como el de ser pacíficos o amantes de la paz. 
Es importante remarcar en estos aspectos porque muestran que la xenofobia no tiene solo condicionantes coyunturales como efectivamente los tiene sino también estructurales. Éstos se atenúan o exacerban en función de situaciones concretas. Es nuestra opinión que en Costa Rica una causa que podríamos catalogar como coyuntural es la que deriva de los cambios que ha sufrido el país desde la década de los 80 del siglo XX, a raíz de la implementación del llamado modelo neoliberal que ha tenido como consecuencia el crecimiento de una brecha social que se ensancha paulatina pero constantemente. En el país se han perfilado por lo menos dos países distintos, con posibilidades, aspiraciones y valores cada vez más divergentes que han empezado a enfrentarse cada vez más agresivamente y cuya primera gran expresión de fuerza se vio en las elecciones presidenciales del 2018. De esos dos países uno se siente marginado y parece no tener muchas expectativas de abandonar ese lugar, y asume posiciones de revanchismo en contra de quienes parecen tener en sus manos el reino de las oportunidades.

Ese país marginado es manipulable sobre todo cuando se le hace creer 6 que los beneficios que se le niegan le serán otorgados a quienes se le ha enseñado que son inferiores a él casi “por naturaleza”. Su reacción puede llegar a ser violenta, como se ha podido comprobar.

La xenofobia no es exclusiva de Costa Rica ni mucho menos. Es un fenómeno que conoce expresiones recurrentes y cada vez más frecuentes en todo el mundo, pero eso no excluye identificar los detonantes particulares que se encuentran en su base en cada circunstancia específica con el fin de tratar de paliar sus expresiones. Como también puede corroborarse con cierta facilidad, el fenómeno tiene siempre componentes estructurales y coyunturales, y cada uno debe ser tratado de acuerdo a su especificidad. 
¿Cómo los discursos sobre las identidades nacionales han incidido en la integración o desintegración de bloques en América Latina, sean estos politicos o económicos, como el Sistema de Integración Centroamericana (SICA), la Alianza Bolivariana para los Pueblos de Nuestra América (ALBA), la Alianza del Pacífico, por ejemplo?

Desde un punto de vista histórico, en general, los discursos relativos a las identidades nacionales en América Latina se han armado precisamente para justificar las artificiales separaciones entre los distintos países que se fueron conformando posteriormente a la independencia, en el siglo XIX.

Digo "generalmente" aunque tal vez debería decir con mayor propiedad especialmente, porque tales discursos tienen antecedentes incluso en el período Colonial, cuando españoles americanos o criollos se sienten pertenecientes a sus respectivas regiones, en un proceso contradictorio en el que quieren ser como los españoles peninsulares pero, al mismo tiempo, se identifican con la tierra en donde han nacido.

Son precisamente esos sentimientos, pero además intereses -económicos, políticos-de los criollos los que determinarán que se concrete en el siglo XIX la separación en países distintos los restos del antiguo Imperio Español. Las diferencias regionales, geográficas y de otro tipo no eran suficientes para justificar la parcelación, por lo que los criollos -clase dirigente del proceso de independencia - transformados en oligarquía terrateniente se tuvieron que dar a la tarea de imaginar diferencias que distinguían a unos países de otros.

La separatista que desembocó en la creación de los actuales países latinoamericanos fue la que prevaleció frente a la unionista o la federalista que, sin embargo quedaron como sustrato o como herencia ideológica que a la larga, se fue transformando en utopía y asociándose con proyectos políticos de sectores que podríamos caracterizar como contestatarios o alternativos 
vinculados a lo popular llenando mucho de su contenido conceptual con puntos de vista vinculados con nuevas condiciones políticas y sociales.

Es así como, a partir de finales del siglo XIX a la idea de unión latinoamericana se le agrega el antiimperialismo. Esto sucede en el contexto de la creciente presencia militar, económica y cultural en América Latina de los Estados Unidos. La unión devenida hacia mediados del siglo XX en integración se considera desde entonces, por parte de fuerzas progresistas o de izquierda, en base para un desarrollo autónomo, independiente de lo que se consideran los prevalecientes intereses estadounidenses en la región.

El ALBA fue desde un inicio proyecto de los gobiernos de la ola progresista latinoamericana de la primera década del siglo XXI y, por lo tanto, se entendió en el sentido antes apuntado: como una propuesta latinoamericanista orientada al apoyo mutuo excluyente de los Estados Unidos y de su proyecto, el ALCA.

Se inspira en una tradición "soterrada" -para usar el término utilizado por los sandinistas en la década de los 80 para referirse a la herencia de Sandino- que solo con la asociación de nuevos grupos sociales al poder del Estado puede transformarse en hegemónica e impulsarse como política.

$\mathrm{Su}$ futuro está asociado a la permanencia en el poder de tales grupos; si estos son desplazados del poder el proyecto se deteriora o tiende a desaparecer. Es lo que está sucediendo con el ALBA en la actualidad y lo que se puede decir de cualquier otro proyecto integracionista en América Latina. En el caso concreto del SICA, este siempre ha estado bajo la égida de empresarios muy dependientes del capital foráneo, especialmente norteamericano y de compañías transnacionales asociadas a ese país. En este contexto, al SICA no le interesa la parte cultural que pueda eventualmente apoyar una identidad centroamericana o centroamericanista. Mientras siga comandado por estos grupos, sectores y clases, prevalecerán los enconos nacionalistas. 